\title{
Pick Your Poison: Benzalkonium Chloride and Copper Enable Nanocellulose Derivatives to Form Antimicrobial Properties Against a Spectrum of Microorganisms
}

\author{
Matthew J. Winans ${ }^{\text {b }}$, Jennifer E.G. Gallagher ${ }^{\mathrm{b}}$, Jacek Jaczynski ${ }^{\mathrm{c}}$, and Gloria Oporto ${ }^{\mathrm{a}}{ }^{*}$ \\ a: Division of Forestry and Natural Resources, $b$ : Department of Biology, $c$ : Division of Animal and \\ Nutritional Sciences, West Virginia University, Morgantown, WV 26506 USA. \\ *Corresponding author: Gloria Oporto gloria.oporto@mail.wvu.edu
}

Keywords: Antimicrobial properties; Benzalkonium chloride; Lignin; TEMPO nanofibrillated cellulose

Abbreviations: TEMPO - 2,2,6,6-tetramethylpiperidine-1-oxyl molecule, TNFC-TEMPO nanofibrillated cellulose, NFC - nanofibrillated cellulose, CMC - carboxymethyl cellulose, BZK - benzalkonium chloride, PVA - polyvinyl alcohol

\section{ABSTRACT}

TEMPO nanofibrillated cellulose (TNFC), nanofibrillated cellulose (NFC), carboxymethyl cellulose (CMC), and lignin were used as templates for the addition of two well-known antimicrobial substances, benzalkonium chloride (BZK) and copper. The resulting hybrid of cellulose and antimicrobial materials were analyzed for biocidal activity in three separate products. Assays of the nanocellulose-antimicrobials were assayed for viability against Escherichia coli in suspension, against Saccharomyces cerevisiae on PVA plastic, and against bacillus lincheniformis in paper additives. Instant biocidal activity was achieved with a minimal inhibitory concentration of $0.116 \mathrm{M}$ BZK-TNFC hybrid suspension. BZK-Lignin and BZKCMC suspensions demonstrated increased antimicrobial activity with longer exposure times during a 24hour exposure which completely inhibited the bacteria. BZK was slowly released into the suspension, a desirable trait for long-term antimicrobial activity. PVA plastic incorporated with BZK/Cu-nanocellulose scaffolds created solid films that completely inhibited yeast growth by 270 seconds. Interestingly, ligninBZK PVA films counteracted each other and showed no biocidal activity at all. The multiple combinations of nanocellulose and biocidal agents in the surface viability assay demonstrates the importance of synergy between both components in designing nanocellulose antimicrobials. TNFC-Cu was more suited to inhibit growth in paper than NFC-Cu as seen in a zone of inhibition assay. The most potent biocidal material in PVA was NFC-BZK. Here we show the diversity of the cellulosic derivatives and their impact on the antimicrobial additive. We employed a variety of assays to assess to biocidal of these nanoparticles against three species of bacteria and yeast relevant to food packaging and medical fields. From our study, there are many factors that play a role in the design of antimicrobial materials; cellulose derivative scaffold, antimicrobial agent, type of final material in which to be incorporated, target organism, and duration of application. 


\section{INTRODUCTION}

Cellulose has an extensive range in industrial and technological applications that includes the production of paper, household commodities, and development of advanced biomedical tissue engineering scaffolding (Haroun et. al 2009; Yang et. al 2011). The natural abundance, innate nontoxicity, and biodegradability of cellulose makes it a desirable material, but its lack of antimicrobial properties limit their spectrum of application. Many research groups have focused on improving this specific shortcoming by affixing additional molecules that exhibit antimicrobial behavior. Cellulose is a suitable template for nanomaterials because of its large number of functional groups available for chemical modification (Cai et. al 2009; Shin et. al 2007). Nanocellulose production has primarily centered on the incorporation of nonorganic metallic nanoparticles (Diez et. al 2011; Silva et. al 2014; Zhong et al. 2013). The untapped potential of organic compounds such as benzalkonium chloride (BZK) and copper remains unexplored in nanocellulose science and may prove appropriate for biomedical applications.

Benzalkonium chloride (BZK) is an organic salt that is well known for its biocidal activity against bacteria, fungi, protozoa, and some viruses. BZK is positively charged and acts as a cationic surfactant interacting with the negatively charged cell wall and the plasma membranes. This interaction permits leakage of small molecules, degradation of biomolecules, and eventual lysis facilitating cell death (Krishnan et. al., 2018). BZK is stable in a wide range of conditions and is commonly used as a bioactive agent in many pharmaceutical, personal care, and disinfectant consumer products. Unlike many other organic molecules with similar biocidal properties, BZK's toxicity to humans is low and allows for topical medicinal application (Thorsteinsson et. al 2003). BZKs effectiveness in the synthesis of antimicrobial cellulose biocomposites has been established in previous research. The incorporation of a chitosan-BZK complex into microfibrillated cellulose increased strength and antimicrobial properties of sodium alginate films, commonly utilized for food packaging applications (Liu et. al 2013). Additionally, through the immersion of freeze-dried bacterial cellulose films in a BZK solution, the resulting cellulose-BZK hybrid films effectively inhibited Staphylococcus aureus and Bacillus subtilus for 24 hours at low concentrations (Wei et. al 2011).

This research took a novel approach to the hybridization of cellulose and BZK by utilizing TEMPO (2,2,6,6-tetramethylpiperidine-1-oxyl)-oxidized nanofibrillated cellulose (TNFC) and carboxymethyl cellulose (CMC) to entrap BZK molecules in a hybrid suspension. TNFC and CMC are distinguished from other cellulose derivatives by their high surface area ratio and the presence of converted carboxyl groups that aid in the physical binding of organic molecules (Isogai et. al 2011). The antimicrobial properties of the resulting suspensions were tested against a gramnegative bacteria, Escherichia coli. Furthermore, these cellulose scaffolds were complexed with copper as a paper/fiber additive and were tested against gram-positive bacteria, bacillus lincheniformis, as a representative for nosocomial infections and biofilm formation in biomedical applications. Lastly, antimicrobial properties of the nanocellulose-Cu/BZK embedded in polyvinyl alcohol (PVA) films were assayed through surface viability against Saccharomyces cerevisiae as a representative of spoilage in packaged foods (Jiang, et. al 2016). Solid cellulose-BZK film 
material was obtained through a solvent exchange and freeze-drying procedure, previously detailed (Zhong et. al 2015). Films were characterized using inverse gas chromatography (IGC). PVA film synthesis and viability assessment demonstrated the feasibility of nanocelluloseantimicrobial complexes in food packaging and medical applications by providing microbial biocidal activity through a controlled release system.

\section{RESULTS AND DISCUSSION}

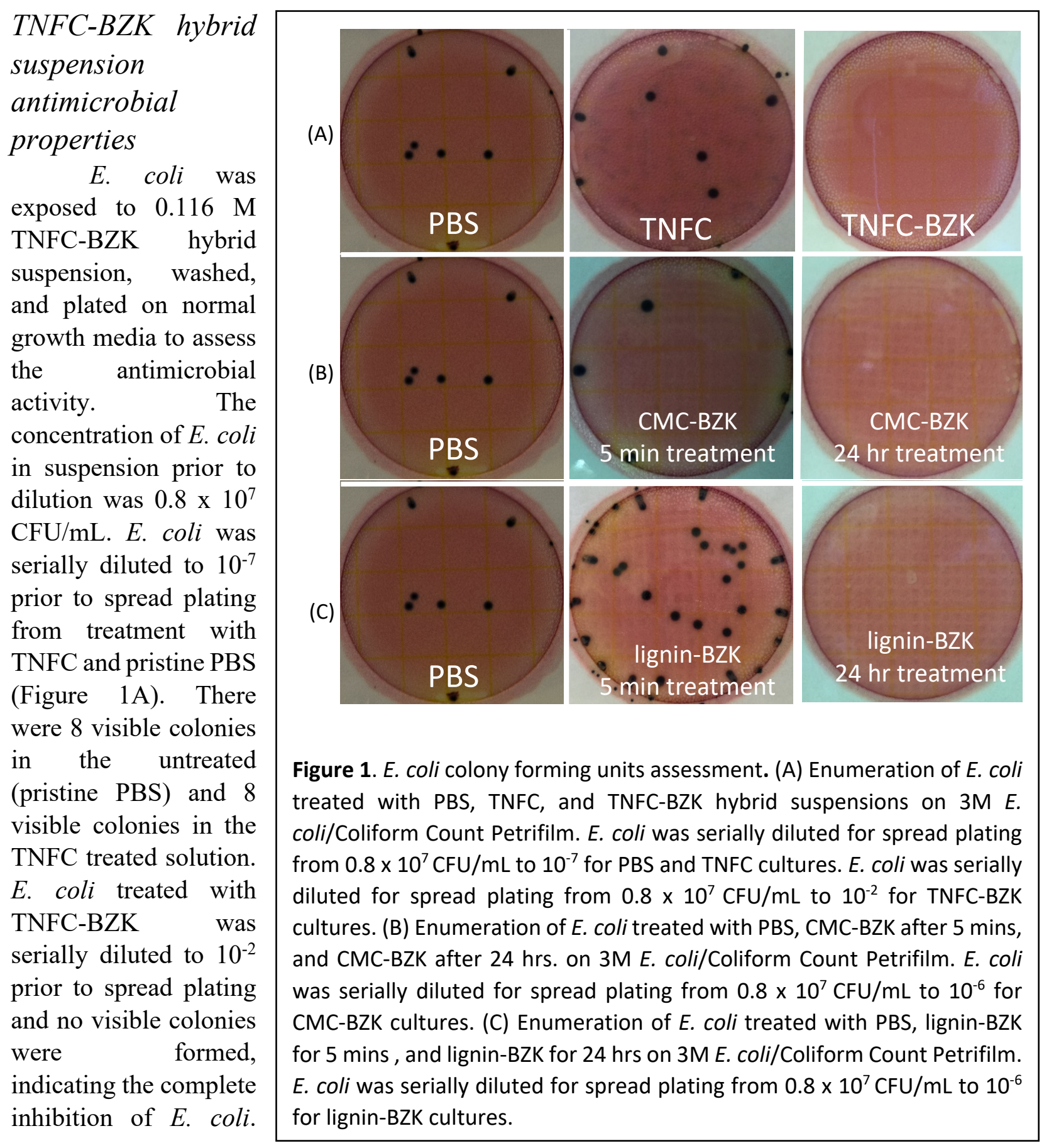


Given the identical amounts of TNFC in both treatments, the antimicrobial action can be attributed to BZK.

\section{CMC-BZK and Lignin-BZK hybrid suspension antimicrobial properties}

To assess the role of different cellulose scaffolds on antimicrobial effectiveness and timeliness, CMC and lignin were used instead of TNFC. Enumeration of E. coli treated with pristine PBS, 0.046 M CMC-BZK suspension for 5-minutes, and 0.046 M CMC-BZK hybrid suspension for 24-hours was conducted (Figure 1B). E. coli was serially diluted to $10^{-7}$ in preparation for spread plating PBS treatment. E. coli was serially diluted to $10^{-6}$ in preparation for spread plating the CMC-BZK timed treatments. When E. coli was treated for 5 min with $0.046 \mathrm{M}$ $\mathrm{CMC}-\mathrm{BZK}, 0.6 \times 10^{6} \mathrm{CFU} / \mathrm{mL}$ was present after treatment. There were no visible colonies after 24 hours, indicating the complete inhibition of $E$. coli at this concentration.

To assess the role of different cellulose scaffolds on antimicrobial effectiveness and timeliness, Lignin-BZK suspensions were used instead of TNFC. Enumeration of E. coli treated with pristine PBS, $0.046 \mathrm{M}$ Lignin-BZK suspension for a 5 minutes, and Lignin-BZK suspension for 24 hours was conducted (Figure 1C). E. coli was serially diluted to $10^{-7}$ in preparation for spread plating PBS treatment. E. coli was serially diluted to $10^{-6}$ in preparation for spread plating the lignin-BZK timed treatments. When the E. coli was treated for 5 minutes with lignin-BZK hybrid suspension, $3.1 \times 10^{6} \mathrm{CFU} / \mathrm{mL}$ were detected, while there were no visible colonies at $10^{-6}$ for CMC-BZK cultures after 24-hrs of treatment. These results, in combination with results from $\mathrm{CMC}$ testing, indicate an increase in antimicrobial properties associated with longer exposure times.

\section{Quantification of surface viability}

To assess the antimicrobial abilities of the nanocellulose-biocidal complexes in PVA plastic against yeast, changes in $S$. cerevisiae viability were recorded. The materials were incorporated into polyvinyl alcohol (PVA) films using a solvent casting method. $S$. cerevisiae RM11, a hardy agricultural isolate, was exposed to the final films. NFC films, complexed with either biocidal agent, were the most potent material with no viable yeast after 30 seconds of exposure while 90 seconds of exposure was required for NFC-Cu (Figure 2). Carboxymethyl cellulose was the least effective cellulose scaffold for acute exposure and second least effective in the overall BZK exposure trials. Lignin-Cu material provided substantial decrease in viability. For BZK additions, lignin resulted in the least effective hybrid material with no effect on viability. The lignin-BZK film caused no cellular death, completely countering the effect of BZK as an antimicrobial agent. More research is needed to determine the rescuing effect observed in this study. TNFC-BZK closely mirrored the highly toxic rate of cellular death of NFC-BZK. When TNFC is coupled with copper, it does not mirror the high toxicity that NFC-Cu does. The difference seen is suggested to be from the synergistic interaction between cellulose scaffold and the biocidal agent.

The difference in synergy between scaffold and biocidal agent suggests that pairing of the scaffold and agent is an important consideration for its intended use as a PVA plastic. Copper generates free radicals through Fenton reactions when it interacts with water and previous work 
demonstrated the antimicrobial activity of $\mathrm{CMC}-\mathrm{Cu}$ hybrid suspensions against $S$. cerevisiae (Rong-Mullins et al. 2017). BZK disrupts the cellular membrane's surface charge and bacterial studies have shown that Pseudomonas aeruginosa copes with benzalkonium chlorides (BAC) in three ways; decreasing porin expression related to BAC, increasing efflux pump activity, and stabilizing the cell surface charge (Krishnan et al. 2018). Thus, these biocidal agents have different modes of action that require consideration when designing antimicrobial cellulose materials.
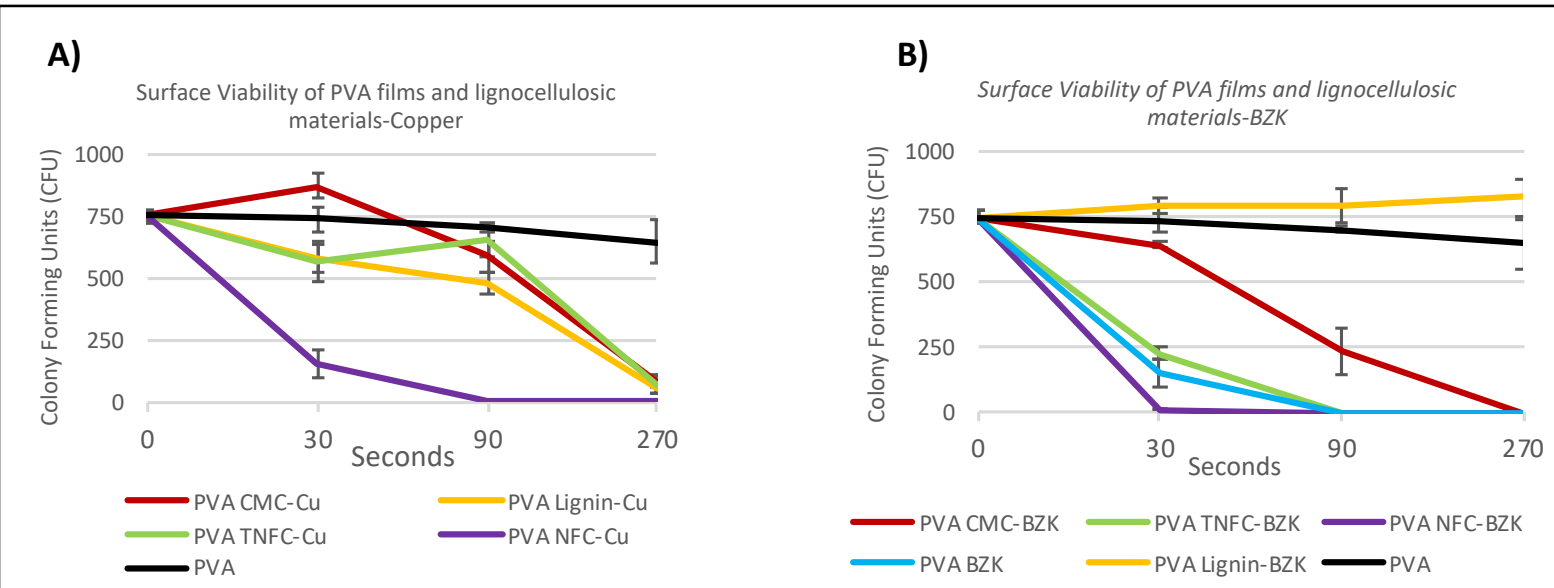

Figure 2. Viability of S. cerevisiae yeast in contact with PVA surfaces embedded with nanocellulosebiocidal materials. Colony forming units were assayed for each surface material in the indicted exposure time in seconds.

\section{Zone of inhibition}

To assess the antimicrobial abilities of these materials incorporated into paper products, a zone of inhibition assay was conducted. Bacillus licheniformis is a bacteria responsible for a range of clinical conditions and food spoilages (Salkinoja-Salonen et al. 1999). TNFC-Cu paper discs created the largest zone of inhibition at $2.72 \mathrm{~cm}$ with a standard deviation of 0.11 followed by $\mathrm{NFC}-\mathrm{Cu}$ at $2.01 \mathrm{~cm}$ with a standard deviation of 0.15 (Figure 3). A student's $\mathrm{T}$ test resulted in $\mathrm{p}$ value of $1.1 \times 10^{-17}$. CMC-Cu discs did not show any zones of inhibition, but several discs showed what appeared to be an immediate

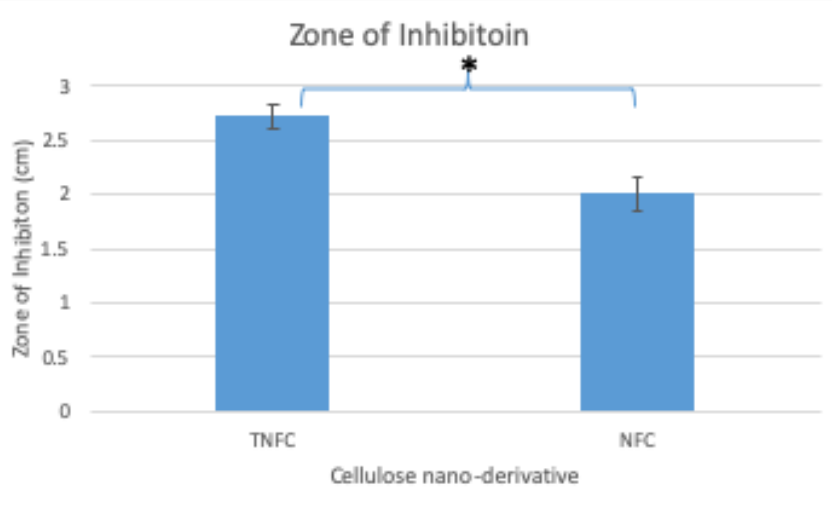

Figure 3. Zone of inhibition for $B$. lincheniformis against cellulose derivatives and copper hybrid materials infused in paper. In the disc diffusion assay, larger zones of inhibition represent increased toxicity of the substance against $B$. lincheniformis. Zone of inhibition measured by their diameter in ImageJ. Students $T$ test performed in Excel, $p=1.1 \times 10^{-17}$. Bacillus lincheniformis (ATCC 14580) on Nutrient Broth after 2 days growth. 
ring of slightly retarded growth at an average diameter of $1.7 \mathrm{~cm}$ with a standard deviation of 0.16 (results not shown). The $\mathrm{CMC}-\mathrm{Cu}$ growth was distinctly different from the inhibition displayed by TNFC-Cu and NFC-Cu materials. The zone of inhibitions of each cellulose derivative was markedly different, which reflects the importance of the variation in cellulose scaffolds while constructing antimicrobial materials.

\section{CONCLUSIONS}

BZK was successfully used as an organic biocide to improve the antimicrobial properties of modified cellulose against bacteria and fungi. The suspensions of CMC-BZK or Lignin-BZK at $0.046 \mathrm{M}$ sufficiently inhibited nonpathogenic E. coli after 24 hours of exposure. Here, we presented evidence of a controlled release mechanism for BZK particles in suspension. The increased antimicrobial properties seen by increasing exposure times supports a controlled release system. BZK complexed with NFC as the scaffold improved the ability to inhibit fungi compared to BZK alone in our surface viability assay. Both BZK and copper result in the eventual lysis of fungi, but through different mechanisms. Inside yeast, the copper released from $\mathrm{CMC}-\mathrm{Cu}$ induces reactive oxygen species (ROS) causing free radical damage to biological molecules including protein, DNA, and lipids (Rong-Mullins, et al. 2017). BZK is an amphiphilic molecule and interaction with cellular membranes induces membrane disorganization, leakage of intracellular material, and degradation of nucleic acids/proteins leading to cell death. Copper is an important fungicide and through repeated exposure many agricultural yeast strains are copper tolerant (RongMullins, et al. 2017). The most common mechanism of copper tolerance is a genomic amplification of the CUP1 locus (Karin et al. 1984). The CUP1 gene encodes a metallothionein that binds excess copper, preventing the production of free radicals (Karin et al. 1984). It is unknown if yeast can also become BZK resistant. In bacteria, mutations that reduce the plasma membrane's negative charge and increase efflux pumps increase resistance during long exposures to sublethal levels (Krishnan et al. 2018). The nearly immediate inhibition of all yeast to the NFC-BZK films reduce the risk of selecting resistant pathogens during normal use. Hybrid suspension added to PVA films would increase the value and antimicrobial properties for food packaging applications. From our study, there are many factors that play a role in the design of antimicrobial materials; cellulose derivative scaffold, antimicrobial agent, type of final material in which to be incorporated, target organism, and duration of application.

\section{METHODS \& MATERIALS}

\section{Materials}

TNFC gel (0.96 wt. \% TNFC) was supplied by the Forest Product Laboratory, Madison, WI. PVA (99-100\% hydrolyzed, approximate molecule weight 86000) obtained from Acros Organics, USA. Sterile trypticase soy broth (TSB) was purchased from Becton-Dickinson, USA. E. coli/Coliform Count Petrifilm Plates obtained from 3M, USA. Butterfield phosphate buffer obtained from Hardy Diagnostics, USA. Escherichia coli DH5 $\alpha$ (hereafter called E. coli) was 
purchased from Invitrogen Inc., USA. Sodium carboxymethyl cellulose (CMC) (average molecular weight: 90,000) obtained from Sigma Aldrich, USA. Ethanol (reagent alcohol, specially denatured alcohol formula 3A $95 \%$ and Isopropyl alcohol $5 \%$ by volume) obtained from Sigma Aldrich, USA. Tert-butanol (99.5\%, for analysis) obtained from Acros Organic, USA. Phosphate buffer solution (PBS) obtained from Hardy Diagnostics, Santa Maria, CA, USA. Deionized water was used throughout the study. S. cerevisiae strain, RM11, is derived from an agricultural isolate (Mortimer and Polsinelli 1999). Yeast were grown in YPD media (1\% yeast extract, $2 \%$ peptone, $2 \%$ dextrose). Yeast extract and peptone obtained from DB Difco. Dextrose obtained from Fischer Scientific.

\section{Methods}

\section{Preparation of hybrid material and hybrid PVA films}

TNFC gel $(15 \mathrm{~mL})$ was dissolved in deionized water $(35 \mathrm{~mL})$. The hybrid suspension was prepared by the addition of $10 \mathrm{~mL} \mathrm{BZK}$ solution $(25 \% \mathrm{w} / \mathrm{w})$ to the TNFC solution under vigorous stirring. The suspension was stirred for an additional two hours to facilitate the dispersion and binding of BZK to the cellulosic backbone.

PVA films were cast by preheating deionized water $(90 \mathrm{~mL})$ to dissolve solid PVA particles $(10 \mathrm{~g})$. The TNFC-BZK hybrid suspension was added dropwise to the PVA solution under vigorous stirring and the resulting mixture was stirred for an additional 2 hours. The PVA was then cast in thin layers into Petri dishes and degassed in a desiccator for 24 hours. After degassing, the films were allowed to rest at room temperature for 12 hours before being transferred to a dry oven $\left(50^{\circ} \mathrm{C}\right)$ for 24 hours.

For CMC and Lignin hybrids, solid cellulosic material was added to deionized water (50 $\mathrm{mL})$ and stirred until fully dissolved. A lower concentration of BZK $(10 \% \mathrm{w} / \mathrm{w})$ was added to both solutions due to the limited availability of CMC to complex with BZK. All other hybrid suspensions and films were prepared with the same procedure used for TNFC suspensions detailed above.

\section{Antimicrobial testing of hybrid suspensions}

E. coli was cultured in TSB media for $24 \mathrm{hrs}$. at $37^{\circ} \mathrm{C}$ with agitation set to $150 \mathrm{rpm} .1 \mathrm{~mL}$ aliquots of the saturated culture were used for antimicrobial testing. $1 \mathrm{~mL}$ of saturated E. coli culture was treated by addition to $9 \mathrm{~mL}$ liquid suspension, thoroughly mixed, and then treated for 5 min. Enumeration occurred after a ten-fold serial dilution and spread plating (Black et. al 2006). $1 \mathrm{~mL}$ of the $E$. coli/hybrid suspension was added to $9 \mathrm{~mL}$ PBS and homogenized. Subsequent serial dilutions were performed in PBS. $1 \mathrm{~mL}$ aliquots of each dilution were enumerated using $3 \mathrm{M}$ Petrifilm E. coli counting plates after incubation at $35^{\circ} \mathrm{C}$ for 48 hours.

\section{Surface viability testing of PVA-BZK hybrid films}

The BZK-cellulose PVA films were tested for antimicrobial properties against the budding yeast, Saccharomyces cerevisiae strain RM11, using a surface viability protocol. Saturated overnight cultures of yeast were spun at $3000 \mathrm{rpm}$ for 3 minutes, washed in phosphate-buffered 
saline (PBS) and resuspended in PBS solution. Optical density $600 \mathrm{~nm}$ readings were taken using a Thermo Spectronic model GENESYS 10 UV. Using separated pipette tips, 29,000 S. cerevisiae cells in $20 \mu \mathrm{l}$ were deposited on the surface and spread across the $1 \mathrm{~cm}^{2}$ PVA film surface. PVA films were prewashed in ethanol and air-dried in a chemical fume hood. Data was collected for exposure times of 5, 60, 120, and 300 seconds. Additionally, the untreated control was labeled as timepoint 0 . After the designated exposure times, $10 \mathrm{ml}$ of PBS was used to wash the coupon and harvest all cells into a $50 \mathrm{ml}$ centrifuge tube. The resultant solution with coupon was vortexed and the coupon was discarded. $50 \mu \mathrm{l}$ of the treated cells/PBS suspensions were plated onto YPD. Each plate was incubated at $30^{\circ} \mathrm{C}$ for $48 \mathrm{hr}$. and then colony forming units (CFU) were counted manually by eye.

\section{Zone of inhibition}

Cellulose-copper materials were embedded via filter paper application and allowed to air dry. A hole punch, producing $1 / 4$ inch diameter discs, was used. Nutrient broth $(0.1 \%$ beef broth, $0.2 \%$ yeast extract, $0.5 \%$ peptone, $0.5 \% \mathrm{NaCl}$ ) was used to culture Bacillus lincheniformis (ATCC 14580). Overnight cultures were grown to saturation and diluted by optical density $600 \mathrm{~nm}$ to 0.5 and aseptically spread onto nutrient broth plates. Once dried, the punch-out discs were then placed in triplicate on each inoculated plate. The plate was cultured for 2 days at $37^{\circ} \mathrm{C}$. Pictures were taken after growth had occurred. Pictures were analyzed with ImageJ and each disc's zone of inhibition was measured twice, totaling 6 measurements per treatment. The average and the standard deviation were calculated.

\section{ACKNOWLEDGEMENTS}

Funds for this project were provided by USDA-NIFA Grant No. 2013-34638-21481 "Development of novel hybrid cellulose nanocomposite film with potent biocide properties utilizing low quality Appalachian hardwoods". MJW was supported by The National Science Foundation's grant “IGERT: REN@WVU - Research and Education in Nanotoxicology at West Virginia University", award number 1144676. MJW conducted yeast surface viability and zone of inhibition experiments. Oliver Lin assisted in the viability studies on yeast. JJ \& GO conducted $E$. coli studies.

\section{REFERENCES}

Black, J. L., \& Jaczynski, J. (2006). Temperature Effect on Inactivation Kinetics of Escherichia coli O157:H7 by Electron Beam in Ground Beef, Chicken Breast Meat, and Trout Fillets. Journal of Food Science, 71, 6.

Cai, J., Kimura, S., Wada, M., \& Kuga, S. (2009). Nanoporous cellulose as metal nanoparticles support. Biomacromolecules, 10, 1, 87-94. 
Díez, I., Eronen, P., Österberg, M., Linder, M. B., Ikkala, O., \& Ras, R. H. (2011). Functionalization of nanofibrillated cellulose with silver nanoclusters: fluorescence and antibacterial activity. Macromolecular Bioscience, 11, 9, 1185-91.

Haroun, A. A., Gamal-Eldeen, A., \& Harding, D. R. (2009). Preparation, characterization and in vitro biological study of biomimetic three-dimensional gelatin-montmorillonite/cellulose scaffold for tissue engineering. Journal of Materials Science. Materials in Medicine, 20, 12, 2527-40.

Isogai, A., Saito, T., \& Fukuzumi, H. (2011). TEMPO-oxidized cellulose nanofibers. Nanoscale, 3, 1, 71-85.

Jiang, C., Oporto, G. S., Zhong, T., \& Jaczynski, J. (2016). TEMPO nanofibrillated cellulose as template for controlled release of antimicrobial copper from PVA films. Cellulose, 23, 1, 713-22.

Karin, M. et al. (1984). Primary structure and transcription of an amplified genetic locus: The CUP1 locus of yeast. Proceedings of the National Academy of Science, U. S. A. 81, 33741

Krishnan, R. et al. (2018). Genomic and Transcriptomic Insights into How Bacteria Withstand High Concentrations of Benzalkonium Chloride Biocides. Applied Environmental Microbiology, 84, e00197-18.

Liu, K., Lin, X., Chen, L., Huang, L., Cao, S., \& Wang, H. (2013). Preparation of microfibrillated cellulose/chitosan-benzalkonium chloride biocomposite for enhancing antibacterium and strength of sodium alginate films. Journal of Agricultural and Food Chemistry, 61, 26, 6562-67.

Mortimer, R. \& Polsinelli, M. (1999). On the origins of wine yeast. Research in Microbiology, 150, 199-204.

Salkinoja-Salonen, M. S. et al. (1999). Toxigenic strains of Bacillus licheniformis related to food poisoning. Applied Environmental Microbiology, 65, 4637-45.

Shin, Y., Bae, I.-T., Arey, B. W., \& Exarhos, G. J. (2007). Simple preparation and stabilization of nickel nanocrystals on cellulose nanocrystal. Materials Letters, 61, 3215-17.

Silva, F. M., Pinto, R. J. B., Daniel-da-Silva, A. L., \& Trindade, T. (2014). Cationic release behavior of antimicrobial cellulose/silver nanocomposites. Cellulose, 21, 5, 3551-60.

Thorsteinsson, T., Másson, M., Kristinsson, K. G., Hjálmarsdóttir, M. A., Hilmarsson, H., \& Loftsson, T. (2003). Soft antimicrobial agents: synthesis and activity of labile environmentally friendly long chain quaternary ammonium compounds. Journal of Medicinal Chemistry, 46, 19, 4173-81. 
Rong-Mullins, X. et al. (2017). Proteomic and genetic analysis of the response of S. cerevisiae to soluble copper leads to improvement of the antimicrobial function of cellulosic copper nanoparticles. Metallomics, 9, 1304-15.

Wei, B., Yang, G., \& Hong, F. (2011). Preparation and evaluation of a kind of bacterial cellulose dry films with antibacterial properties. Carbohydrate Polymers, 84, 1, 533-38.

Yang, C., Gao, C., Wan, Y., Tang, T., Zhang, S., \& Dai, K. (2011). Preparation and characterization of three-dimensional nanostructured macroporous bacterial cellulose/agarose scaffold for tissue engineering. Journal of Porous Materials, 18, 5, 54552.

Zhong T., Oporto G.S., Jaczynski J. Tesfai A. and J. Armstrong. (2013). Antimicrobial properties of the hybrid copper nanoparticles-carboxymethyl cellulose. Wood Fiber Sci. 45(2) 1-8.

Zhong, T., Oporto, G. S., Peng, Y., Xie, X., \& Gardner, D. J. (2015). Drying cellulose- based materials containing copper nanoparticles. Cellulose, 22, 4, 2665-81. 